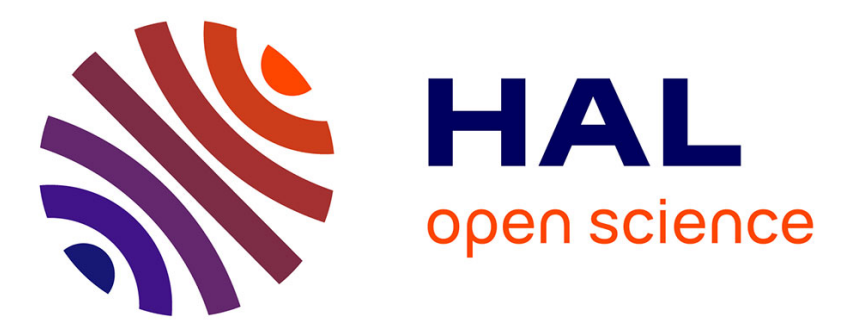

\title{
Comportement thermomécanique d'un béton ordinaire en compression cyclée: expérience et modélisation
}

\author{
Vincent Huon, Olivier Maisonneuve, Bruno Cousin
}

\section{To cite this version:}

Vincent Huon, Olivier Maisonneuve, Bruno Cousin. Comportement thermomécanique d'un béton ordinaire en compression cyclée: expérience et modélisation. Comptes rendus de l'Académie des sciences. Série IIb, Mécanique, 2001, 329, pp.889-896. 10.1016/S1620-7742(01)01415-5 . hal-02457401

\section{HAL Id: hal-02457401 \\ https://hal.science/hal-02457401}

Submitted on 28 Jan 2020

HAL is a multi-disciplinary open access archive for the deposit and dissemination of scientific research documents, whether they are published or not. The documents may come from teaching and research institutions in France or abroad, or from public or private research centers.
L'archive ouverte pluridisciplinaire HAL, est destinée au dépôt et à la diffusion de documents scientifiques de niveau recherche, publiés ou non, émanant des établissements d'enseignement et de recherche français ou étrangers, des laboratoires publics ou privés. 


\title{
Comportement thermomécanique d'un béton ordinaire en compression cyclée : expérience et modélisation
}

\author{
Vincent HUON $^{a}$, Olivier MAISONNEUVE ${ }^{b}$, Bruno COUSIN $^{c}$
}

${ }^{a}$ Laboratoire de mécanique et génie civil, UMR 5508 CNRS-UMII, Université Monpellier II, c.c. 81, place Eugène Bataillon, 34095 Monpellier cedex 05, France

b Laboratoire de mécanique et génie civil, UMR 5508 CNRS-UMII, Université Monpellier II, c.c. 48, place Eugène Bataillon, 34095 Monpellier cedex 05, France

${ }^{c}$ Laboratoire de mécanique et génie civil, UMR 5508 CNRS-UMII, Université Monpellier II, c.c. 34, place Eugène Bataillon, 34095 Monpellier cedex 05, France

Courriel : huon@Imgc.univ-montp2.fr; maisonneuve@Imgc.univ-montp2.fr; cousin@Imgc.univ.-montp2.fr

Résumé. Les faibles phénomènes thermiques apparaissant lors d'essais de compression sur un béton ordinaire ont pu être observés par thermographie infrarouge. Pour leur modélisation et leur interprétation, il est proposé une extension thermomécanique du modèle élastique endommageable de M. Frémond et B. Nedjar. Elle permet un dialogue intéressant entre les résultats expérimentaux et ceux obtenus par simulation numérique.

rhéologie / béton / thermographie infrarouge / endommagement / modélisation /couplages thermomécaniques

\section{Thermomechanical behaviour of a plain concrete in compressive test: experimentation and modelling}

\begin{abstract}
plain concrete during a compressive test. A modelling of the thermomechanical behaviour of this materials is proposed as an extension of M. Frémond and B. Nedjar's model. It permits an interesting dialog between the experimental results and those obtained by a numerical simulation of a simple compressive test.

rheology / concrete / infrared thermography / damage / modelling / thermomechanical coupling
\end{abstract}

\section{Abridged English version}

Regarding plain concrete as a damaging elastic material, this paper presents experimental and modelling results obtained during compressive tests.

Infrared thermography observations permit to show thermal phenomena occurring on a plain concrete during a quasi-static compressive cycle test. We note, figure 1, the superposition of two phenomena: temperature variations in phase with the evolution of the load and a progressive warming-up of the sample

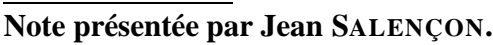


(0.04 $\mathrm{C}$ at the end of the test, figure 2). The first phenomenon could be attributed to the thermoelastic coupling (Huon et al. [1]) and the progressive warming-up to the damade evolution of the sample.

To take into account thermal phenomena associated with the deformation and damage of concrete, we propose to expand the M. Frémond and B. Nedjar's model (Frémond and Nedjar [2,3]). We are led to choose the free energy, $\Psi$, and the pseudo-potential of dissipation, $\Phi$, as equation (1). Constitutive laws and damage variables evolution are described with (2) and (3), respectively. In regard to the temperature variable evolution, the linearized form (5) of the local heat conduction equation (4) gives it.

Six loading-unloading cycles in compression piloted in deformation have been simulated.

Figure 3 gives the stress-strain answer of the material and figure 4 the evolution of the damage with time. We recover a classical behaviour for the concrete requested in compression.

The thermal behaviour is characterized by the evolution with time of the temperature $\theta$ (figure 9), but also the heat sources $w_{c h}$ (figure 8), corresponding to the intrinsic dissipation $D_{1}$ (figure 5), and to the terms of coupling temperature-damage, noted $T-\beta$ (figure 6$)$, and temperature-deformation, noted $T-\varepsilon$ (figure 7 ).

During a cycle, $D_{1}$ (figure 5 ) and $T-\beta$ (figure 6 ) are nonzero if the damage grows ( $\beta_{c}$ decreases) to the load, the threshold of damage being exceeded. The maximal values of $D_{1}$ and $T-\beta$ decrease with the passing of the cycles because of the increase of the damage threshold. We note that $D_{1}$ is negligible in relation to the other heat sources terms. It is not major in the temperature variations generated by the processes of deformation and damage.

With regard to the coupling terms, we observe, for a given cycle, a 'competition' between $T-\beta$ (figure 6$)$, that increases with the damage and $T-\varepsilon$ (figure 7 ), that falls when the damage grows. Figure 8 gives, in practice, the result of this competition, $D_{1}$ being negligible.

If we look at the evolution of $\theta$, we note (figure 9) the superposition of two phenomena:

- the linear temperature variations, in phase with the loading-unloading cycles;

- a progressive warming-up of the material.

The first phenomenon results from the competition between $T-\beta$ and $T-\varepsilon$. If we make a zoom of the first cycle, while the evolution of the temperature of the material assuming zero damage, we note successively (figure 10):

- a thermoelastic evolution before the threshold of damage ( $5 \mathrm{~s})$;

- a compensation of $T-\beta$ and $T-\varepsilon$ effects (until $7.5 \mathrm{~s}$ );

- the influence of $T-\beta$ leading to a temperature variation (nonlinear) more important than the one due to the only thermoelastic effect (linear) and, a fortiori, to the thermoelastic effect with damage (nonlinear).

If we note, more, that the thermoelastic effect corresponding to the unloading of the damaged material $\left(E^{\prime}<E\right)$ cannot compensate the heat supply at the loading, the non-return to zero of $\theta$ at the end of the loading is explained. A progressive elevation of $\theta$ results from it at the end of every cycle (second phenomenon).

The superposition of two phenomena (thermoelastic and temperature-damage couplings) gives results qualitatively very similar than those given by experiment. So we can say that modelling seems to valid the proposed interpretation of experimental results.

\section{Introduction}

Considérant les bétons ordinaires comme des matériaux élastiques endommageables, le travail présenté concerne la mise en évidence, la quantification et la modélisation, lors de cycles de compression quasistatiques, des effets thermiques associés au comportement élastique (couplage thermoélastique) et à l'évolution de l'endommagement de ces matériaux. 


\section{Dispositif expérimental et matériau étudié}

Le dispositif comporte une machine d'essais mécaniques couplée à une caméra infrarouge. Il est possible de suivre en parallèle l'évolution des paramètres mécaniques et celle de la carte de température de la face observée de l'éprouvette. Les sollicitations sont des cycles charge-décharge quasi-statiques en compression, à vitesse de déplacement de traverse imposée.

Les essais ont été mis en œuvre sur des éprouvettes parallélépipédiques $\left(40 \times 40 \times 160 \mathrm{~mm}^{3}\right)$ en microbéton composé de sable siliceux (0-2 mm), avec des rapports eau/ciment $(E / C)$ et ciment/sable $(C / S)$ respectivement de 0,5 et 0,3 . La résistance en compression à 28 jours est d'environ $45 \mathrm{MPa}$.

\section{Résultats expérimentaux}

Six cycles charge-décharge ont été réalisés sur les éprouvettes en béton ordinaire, jusqu'à une contrainte de l'ordre de $35 \mathrm{MPa}$, avec une vitesse de traverse de $5 \cdot 10^{-2} \mathrm{~mm} \cdot \mathrm{s}^{-1}$. La figure 1 permet de suivre l'évolution, au cours du temps et en chaque point de l'axe médian de la face observée de l'éprouvette, des variations de température $\left(\theta=T-T_{0}\right)$ en fonction de l'état de chargement mécanique (courbe blanche).

On constate la superposition de deux phénomènes :

- une variation positive de la température, constante le long de l'éprouvette, en phase avec l'évolution de la charge. L'amplitude maximale des variations observées est de $0,08 \mathrm{C}$ pour une contrainte de $35 \mathrm{MPa}$;

- un faible échauffement progressif de l'éprouvette atteignant 0,04 C en fin d'essai (cf. figure 2).

Le premier phénomène a été précédemment identifié dans le cas d'un béton ultra-performant considéré comme très faiblement endommagé au vu des niveaux de chargement (Huon et al. [1]). Il s'agit du couplage thermoélastique. Dans le cas des bétons ordinaires, les contraintes atteintes en fin de chargement sont très proches de celle à rupture. On associe alors l'échauffement progressif, non observé dans le cas d'un béton ultra-performant [1], à une évolution de l'endommagement du matériau.

\section{Une modélisation du comportement thermomécanique d'un béton ordinaire}

Pour modéliser les phénomènes thermiques associés à la déformation d'un béton qui s'endommage, il est proposé une extension thermomécanique du modèle de M. Frémond et B. Nedjar (Frémond et Nedjar [2,3]) restreint au cas de sollicitations de traction ou compression simples. La variable température intervient, en particulier, dans la modélisation des manifestations thermiques associées aux phénomènes de dilatation.

Figure 1. Évolution au cours du temps du profil de température de l'axe médian de l'éprouvette $(1 \mathrm{~s}=5$ images, 256 pixels $=$ $80 \mathrm{~mm}$ ). Courbe blanche en surimpression : évolution de la charge.

Figure 1. Time evolution of the length-wise temperature profile $(1 \mathrm{~s}=5$ images and 256 pixel $=80 \mathrm{~mm}$ ). Surimposed white curve: load evolution.

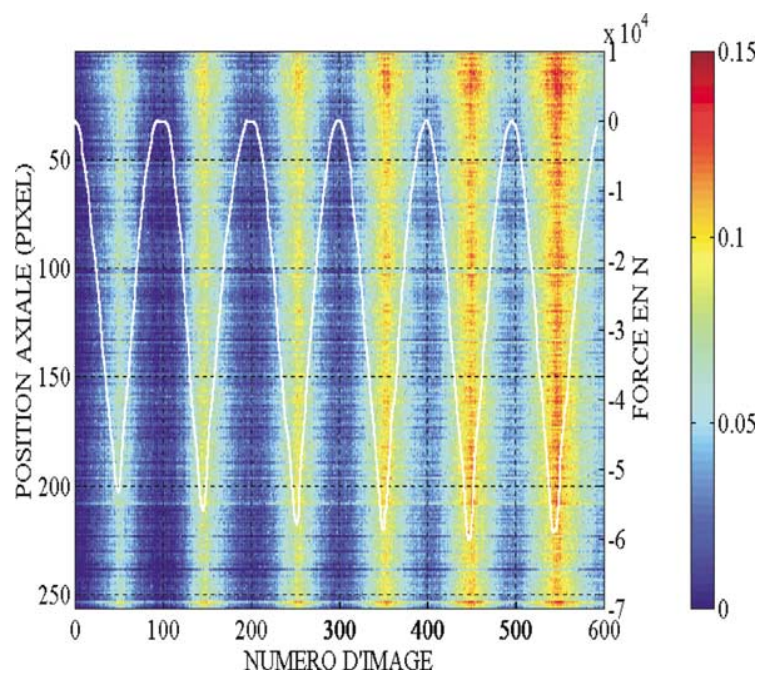




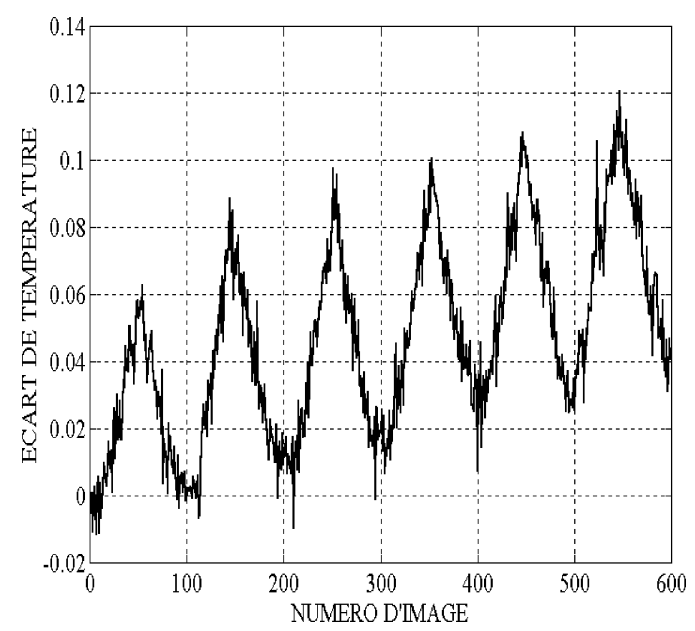

Figure 2. Évolution au cours du temps de la variation de température au point milieu de l'éprouvette.

Figure 2. Time evolution of temperature variation at middle sample point.

Les expressions modifiées de l'énergie libre, $\Psi$, et du pseudo-potentiel de dissipation, $\Phi$, sont alors les suivantes en référence à celles, $\Psi_{f}$ et $\Phi_{f}$, données dans $[2,3]$ :

$$
\Psi=\Psi_{f}-\beta_{t}(3 \lambda+2 \mu) \alpha \theta \operatorname{tr} \varepsilon_{t}+\beta_{c}(3 \lambda+2 \mu) \alpha \theta \operatorname{tr} \varepsilon_{c}-\frac{\rho C}{2 T_{0}} \theta^{2}, \quad \Phi=\Phi_{f}
$$

où $\theta=T-T_{0}$ est l'écart de température, $\alpha$ le coefficient de dilatation thermique linéique, $C$ la chaleur spécifique, $\beta_{t}$ et $\beta_{c}$ les variables d'endommagement respectivement en traction et compression $(\beta=1$ matériau sain, $\beta=0$ matériau ruiné).

Les lois d'état et les lois complémentaires s'écrivent en conséquence, en utilisant les notations de [2,3] avec $i \in\{t, c\}$ :

$$
\begin{aligned}
& \sigma^{r}=\sigma_{f}^{r}-\beta_{c}(3 \lambda+2 \mu) \alpha \theta \operatorname{Id}-\beta_{t}(3 \lambda+2 \mu) \alpha \theta \operatorname{Id}, \quad B_{i}^{r}=\left(B_{i}^{r}\right)_{f}, \quad H_{i}^{r}=\left(H_{i}^{r}\right)_{f} \\
& \sigma^{i r}=H_{i}^{i r}=\left(\sigma^{i r}\right)_{f}=\left(H_{i}^{i r}\right)_{f}=0, \quad B_{i}^{i r}=\left(B_{i}^{i r}\right)_{f}, \quad \sigma=\sigma^{i r}+\sigma^{r}
\end{aligned}
$$

On pose $H_{i}=H_{i}^{r}+H_{i}^{i r}, B_{i}=B_{i}^{r}+B_{i}^{i r}$ résultant de la prise en compte de la puissance des efforts intérieurs associés respectivement à l'endommagement et à son gradient, lors des mouvements microscopiques correspondants [2,3].

Les équations d'évolution quasi-statique pour les variables d'endommagement s'écrivent alors :

$$
\left(E_{1}\left(\beta_{i}, \dot{\beta}_{i}\right)\right)_{f} \ni\left(E_{2}\left(\varepsilon_{i}, \dot{\beta}_{i}\right)\right)_{f}+\eta(3 \lambda+2 \mu) \alpha \theta \operatorname{tr} \varepsilon_{i}
$$

où $\eta=1$ si $i=t, \eta=-1$ si $i=c,\left(E_{1}\right)_{f}$ et $\left(E_{2}\right)_{f}$ s'identifiant aux termes des équations d'évolution de [2,3]. Le terme supplémentaire traduit l'influence de la dilatation thermique.

En adoptant les hypothèses conduisant à la forme classique de l'équation de la chaleur

$$
\rho C \dot{T}-r+\operatorname{div} q=D_{1}+\rho T \frac{\partial^{2} \Psi}{\partial T \partial \alpha_{k}}: \dot{\alpha}_{k}=w_{c h} \quad(k=1, \ldots, n)
$$

on obtient par linéarisation, avec les hypothèses décrites ci-après, l'équation différentielle en $\theta$ suivante :

$$
\rho C\left(\frac{\partial \theta}{\partial t}+\frac{\theta}{\tau_{t h}}\right)=w_{c h}
$$


Les $\alpha_{k}$ sont les $n$ variables d'état (hors la température) retenues, $w_{c h}$ la densité volumique de sources de chaleur, $D_{1}$ la dissipation intrinsèque, $r$ la densité volumique de chaleur fournie par l'extérieur et $q$ le vecteur courant de chaleur. $\tau_{t h}$ est une constante caractéristique des fuites thermiques. Les hypothèses sous-jacentes à la linéarisation sont les suivantes :

(1) le vecteur courant de chaleur $q$ s'écrit $q=-k_{t h} \operatorname{grad} T$ où $k_{t h}$ est le coefficient de conductivité thermique supposé constant et indépendant de la température;

(2) le taux de chaleur volumique fourni par le milieu extérieur $r$ est nul;

(3) les termes convectifs dans la dérivée particulaire de $T$ sont négligés;

(4) les fuites thermiques sont linéaires (Chrysochoos [4]).

\section{Simulation numérique d'un essai de compression}

Pour tester ce modèle, on a considéré une éprouvette parallélépipédique en béton ordinaire, de section carrée, soumise à 6 cycles de charge-décharge en compression pilotés en déformation.

Les efforts volumiques étant négligées, le chargement est supposé uniforme dans l'espace, d'où un endommagement considéré comme tel. De ce fait, le gradient de l'endommagement et le facteur d'influence de l'endommagement pris en compte dans $\Psi_{f}$ n'interviennent pas.

Pour la simulation, on adopte les valeurs suivantes : module d'Young $E=25 \mathrm{GPa}$, coefficient de Poisson $\nu=0,2$, masse volumique $\rho=2,2 \cdot 10^{3} \mathrm{~kg} \cdot \mathrm{m}^{-3}$, température de référence $\mathrm{T}_{0}=298 \mathrm{~K}$, vitesse de déformation $\dot{\varepsilon}_{0}=-1,3 \cdot 10^{-4} \mathrm{~s}^{-1}$.

Les caractéristiques thermiques du béton sont prises dans la littérature, soit : coefficient de dilatation thermique $\alpha=10 \cdot 10^{-6} \mathrm{~K}^{-1}$, chaleur spécifique $C=838 \mathrm{~J} \cdot \mathrm{kg}^{-1} \cdot \mathrm{K}^{-1}$.

La constante de temps caractéristique des fuites thermiques, $\tau_{t h}$, est déterminée par un rapport de proportionnalité avec la conductivité thermique d'un acier : $\tau_{t h}=10^{4} \mathrm{~s}$.

Quant aux coefficients propres au modèle de M. Frémond et B. Nedjar, les valeurs proposées par les auteurs [2,3] ont été reprises.

\subsection{Comportement mécanique}

La figure 3 donne, respectivement, l'évolution de la contrainte au cours du temps et la réponse contraintedéformation du matériau à la sollicitation imposée. On retrouve un comportement classique d'un béton sollicité en compression. A chaque cycle de chargement, le matériau s'endommage un peu plus comme le montre, par ailleurs, la figure 4 donnant l'évolution de l'endommagement en compression au cours du temps. Les phénomènes thermiques mis en jeu étant faibles (cf. résultats expérimentaux et § 2.2), il n’y

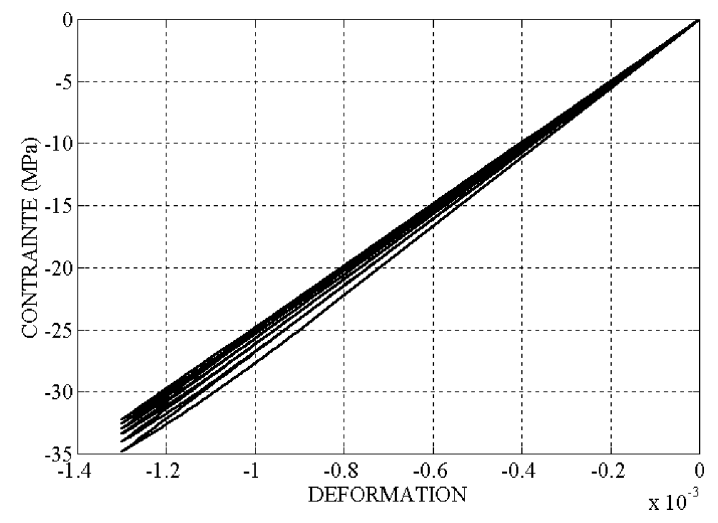

Figure 3. Réponse contrainte-déformation.

Figure 3. Stress-strain relationships.

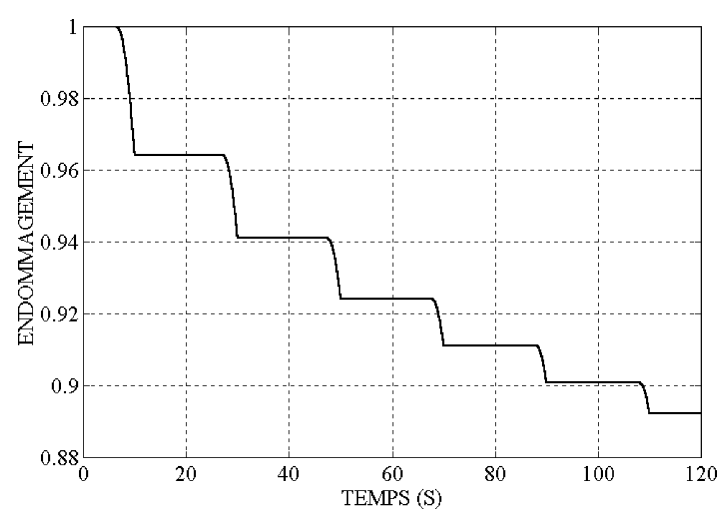

Figure 4. Évolution de l'endommagement.

Figure 4. Damage evolution. 
a pas de différences notoires entre le comportement mécanique isotherme et le comportement mécanique issu de la modélisation.

\subsection{Comportement thermique}

Le comportement thermique, résultant de la déformation du matériau et de son endommagement, est caractérisé par l'évolution au cours du temps de la variation de température (figure 9) et du terme source de chaleur (figure 8 ) résultant de la superposition de la dissipation intrinsèque (figure 5 ), avec les termes de couplage température-endommagement (figure 6 ) et température-déformation (figure 7).

La dissipation intrinsèque (figure 5 ) et le terme de couplage température-endommagement (figure 6 ) sont différents de zéro, dans la phase de chargement d'un cycle, lorsque le seuil d'endommagement est franchi, et donc lorsque l'endommagement croit ( $\beta$ décroissant). Les valeurs maximales de la dissipation intrinsèque et du terme de couplage température-endommagement diminuent au fil des cycles, en raison de l'augmentation du seuil d'endommagement. On constate, de plus, que la dissipation intrinsèque est négligeable par rapport aux autres termes sources de chaleur. Elle ne sera donc pas prépondérante dans les variations de température engendrées par les processus de déformation et d'endommagement.

En ce qui concerne les termes de couplage, on constate, pour un cycle donné, une «compétition » entre le couplage température-endommagement (figure 6 ), qui augmente avec l'endommagement, et le couplage température-déformation (figure 7), qui chute au fur et à mesure que l'endommagement croît. La figure 8 donne essentiellement le résultat de cette compétition, la dissipation intrinsèque étant négligeable. Ces sources de chaleur sont la cause des variations de température (figure 9) que l'on est susceptible d'observer expérimentalement.

Si l'on examine l'évolution des variations de température calculées, sous l'hypothèse de quasiadiabaticité, on constate (figure 9) la superposition de deux phénomènes : des variations de température linéaires par morceaux, en phase avec les cycles charge-décharge, y compris lorsque l'endommagement évolue, et un échauffement progressif du matériau.

Le premier phénomène résulte, comme on l'a vu précédemment pour l'analyse des sources de chaleur, de la compétition entre les termes de couplage température-endommagement et température-déformation.

Si l'on effectue un zoom sur le premier cycle, en faisant figurer en parallèle l'évolution de la température du même matériau s'il ne s'endommageait pas (seul le couplage thermoélastique intervient), on constate (figure 10) :

- une évolution de type thermoélastique avant le seuil d'endommagement (5 secondes);

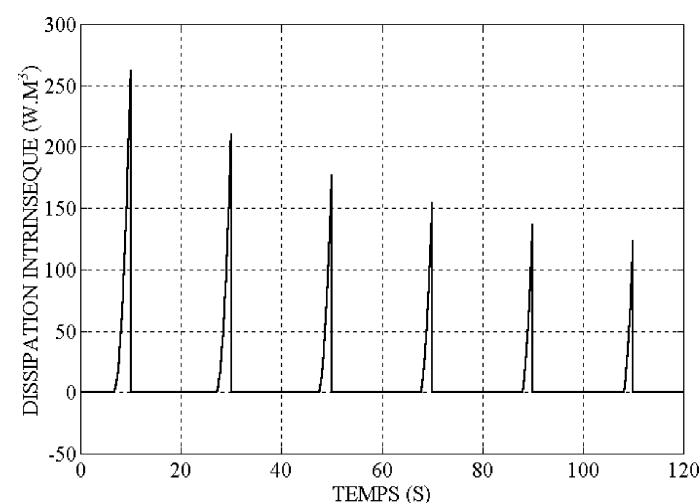

Figure 5. Évolution de la dissipation intrinsèque.

Figure 5. Intrinsic dissipation evolution.

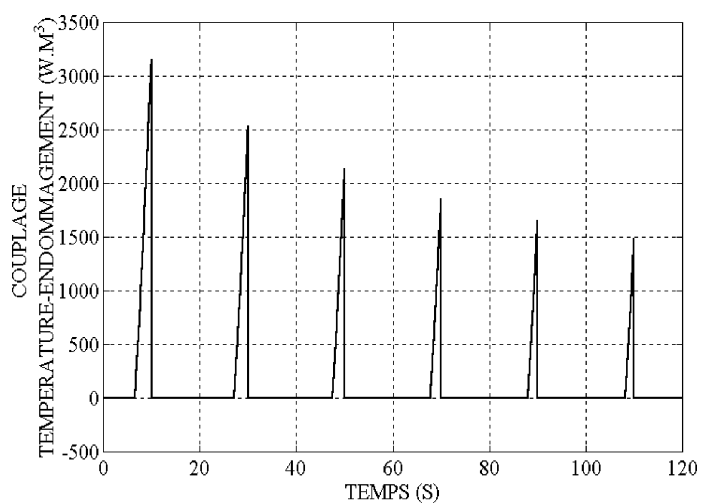

Figure 6. Évolution du terme de couplage température-endommagement.

Figure 6. Temperature-damage coupling term evolution. 


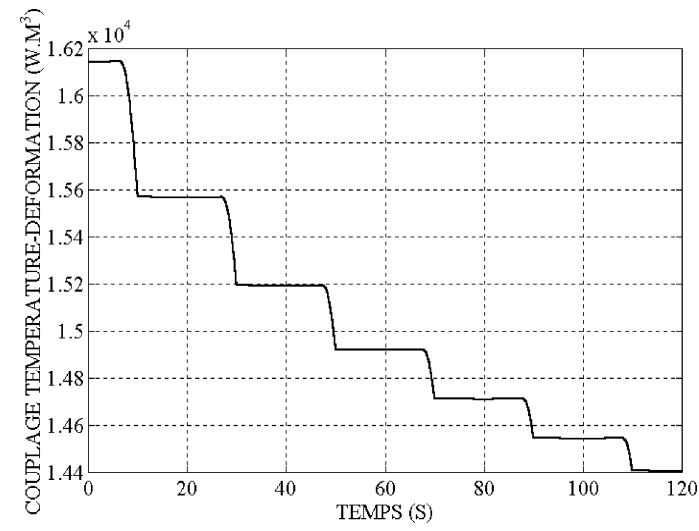

Figure 7. Évolution du terme de couplage température-déformation.

Figure 7. Temperature-deformation coupling term evolution.

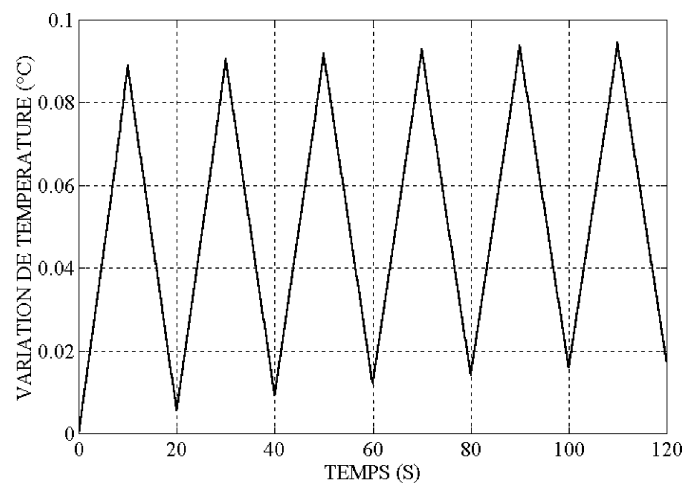

Figure 9. Variation de température.

Figure 9. Temperature variation.

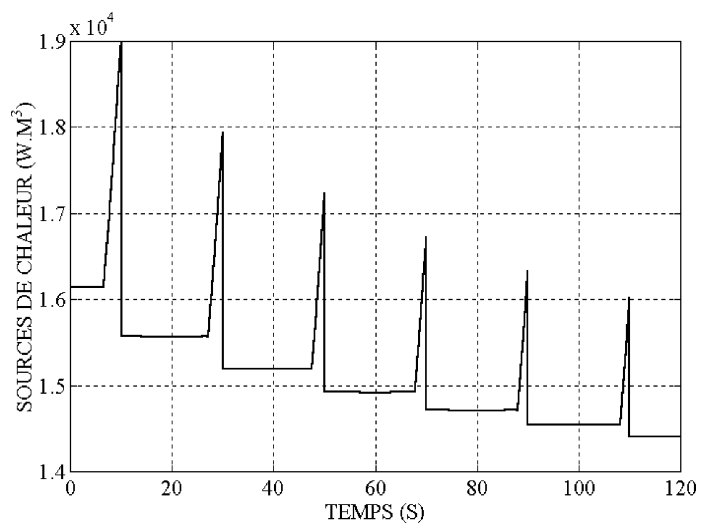

Figure 8. Évolution des sources de chaleur.

Figure 8. Heat sources evolution.

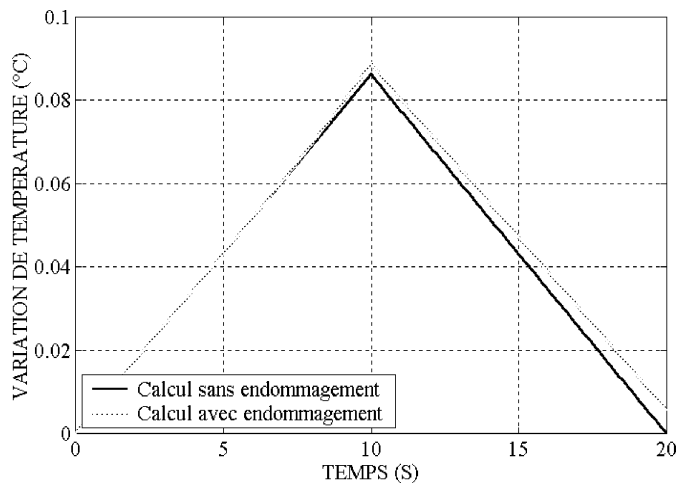

Figure 10. Variation de température pour le $1^{\mathrm{er}}$ cycle de chargement avec et sans endommagement.

Figure 10. Temperature variation for the 1st loading cycle with damage (gray curve) and without (black curve).

- ensuite une compensation des deux termes de couplage température-endommagement et températuredéformation (jusqu'à 7,5 secondes environ);

- puis l'influence prépondérante du terme de couplage température-endommagement conduisant à une variation de température (non linéaire) plus importante que celle due au seul effet thermoélastique (linéaire).

Le non-retour à zéro des variations de température à la fin de la décharge s'explique par le fait qu'à la décharge le refroidissement isentropique $\left(E^{\prime}<E\right)$ ne peut pas compenser l'apport de chaleur à la charge. Il en résulte une élévation progressive de la température à la fin de chaque cycle (deuxième phénomène).

\section{Confrontation expérience/modélisation. Conclusion}

Sous les hypothèses d'isotropie des comportements thermique et mécanique du matériau, il ressort de la modélisation proposée : 
- sur le plan purement mécanique, une étude qualitativement correcte du comportement mécanique d'un béton comme dans [2,3];

- sur le plan thermique, que certains phénomènes sont prépondérants. Les termes de couplage température-endommagement et thermoélastique dominent la dissipation intrinsèque. Les variations de température calculées, inférieures à $0,2 \mathrm{C}$, sont du même ordre de grandeur que celles observées expérimentalement.

Il en résulte une relecture intéressante des résultats expérimentaux obtenus lors des essais cyclés de compression : le phénomène d'échauffement progressif de l'éprouvette apparaît bien, au vu des résultats de la simulation numérique, comme associé à une évolution de l'endommagement. La modélisation et les résultats expérimentaux se confortent mutuellement. Les interprétations adoptées dans notre étude semblent donc judicieuses.

\section{Références bibliographiques}

[1] Huon V., Cousin B., Maisonneuve O., Mise en évidence et quantification des couplages thermomécaniques réversibles et irréversibles dans les bétons sains et endommagés par des cycles gel-dégel, C. R. Acad. Sci. Paris, Série IIb 329 (2001) 331-335.

[2] Frémond M., Nedjar B., Endommagement et principe des puissances virtuelles, C. R. Acad. Sci. Paris, Série II 317 (1993) 857-864.

[3] Frémond M., Nedjar B., Damage, gradient of damage and principle of virtual power, Int. J. Solids Structures 33 (8) (1996) 1083-1103.

[4] Chrysochoos A. Dissipation et blocage d'énergie lors d'un écrouissage en traction simple, Thèse de doctorat d'état, Université Montpellier II, 1987. 